\title{
Health Care Workers Adherence to Infection Prevention Practices and Control Measures: A Case of a Level Four District Hospital in Kenya
}

\author{
Alice W. Gichuhi ${ }^{1, ~}$, Simon M. Kamau ${ }^{2}$, Elijah Nyangena ${ }^{2}$, Z. Ngalo Otieno-Ayayo ${ }^{3}$ \\ ${ }^{1}$ Training Centre, Moi Teaching \& Referral Hospital, Eldoret, Kenya \\ ${ }^{2}$ School of Science \& Technology, Department of Nursing Sciences, University of Kabianga, Kericho, Kenya \\ ${ }^{3}$ School of Science, Technology and Engineering, Rongo University College, Rongo, Kenya

\section{Email address:} \\ aligichuhi@yahoo.com (A. W. Gichuhi), smacharia@kabianga.ac.ke (S. M. Kamau), elijah208@yahoo.com (E. Nyangena), \\ zngalo@ruc.ac.ke (Z. Otieno-Ayayo)
}

\section{To cite this article:}

Alice W. Gichuhi, Simon M. Kamau, Elijah Nyangena, Z. Ngalo Otieno-Ayayo. Health Care Workers Adherence to Infection Prevention Practices and Control Measures: A Case of a Level Four District Hospital in Kenya. American Journal of Nursing Science.

Vol. 4, No. 2, 2015, pp. 39-44. doi: 10.11648/j.ajns.20150402.13

\begin{abstract}
Infection prevention practices and control are critical activities that influence the quality of health care services. This study was conducted to determine the health workers adherence to infection prevention and control policies and procedures at a Level 4 Hospital in Kenya. A descriptive cross-sectional survey design were used. The objectives of the study were to: -Identify existing infection prevention and control policy guidelines at the level four hospital; -Evaluate the implementation of infection prevention and control measures; 3) Identify the barriers to compliance with infection prevention practices and control measures. Data was collected by questionnaire, record review and a focus group discussion. Quantitative data was analyzed by the statistical Package for Social Sciences (SPSS) while information from focus group was analyzed thematically. The results revealed that Health Care Workers (HCWs) had good knowledge on infection prevention practices and control. There were written infection prevention practices and control (IPPC) policy guidelines and high awareness (98.7\%) of the IPPC policy guidelines. The record review showed that there was approximately $6.7 \%$ nosocomial infections rate among hospitalized patients. Barriers to IPPC compliance among the health care workers (HCWs) included frequent shortage of water, inadequate updates on IPPC through continuing professional education and inactive IPPC committee. The study concluded that there was adequate compliance with IPPC, though there were challenges to implementation that needed to be addressed. The study was done in October 2010 to January 2011
\end{abstract}

Keywords: Infection Prevention Practices and Control, Nosocomial Infections, Health Care Workers, Standard Precautions, Adherence, Compliance

\section{Introduction and Literature Review}

Hospital acquired infections (HAIs)/nosocomial infections pose a real and serious threat to both the patients and health care workers. Common pathogens may easily be transmitted through health care workers' hands, equipment, supplies and unhygienic practices. Adherence to infection prevention and control guidelines is critical to improving the quality of hospital care based on their efficacy in reducing the occurrence of infections that compromise patients' outcomes [1]. However, it was uncertain how well the health care workers at level four hospitals in Kenya adhered to infection control and prevention practices.

Inadequate infection prevention and control in hospitals was shown to have a number of consequences. These included increased bed occupancy and a strain on drugs and other scarce hospital resources. Lack of adherence to infection control leads to drugs resistance by micro-organism, lengthens time spent by admitted patients in the hospital. Further this translates into high costs to the hospital and the patient as well as increased social suffering for the patient and family [2].

A significant proportion of in-patient care activities are done by nurses who comprise majority workers in the health care facilities. These activities include administering 
parenteral medications, cleaning, hygiene, feeding, handling of patients' beddings, dressing of wounds and giving medications [3]. Therefore, they are constantly exposed to Health Care Acquired Infections (HCAI) such as Tuberculosis, Human Immune Virus and Acquired Immune Deficiency Syndrome (HIV/AIDS), Hepatitis B, diverse bacterial infections among others. Strict adherence to infection prevention protocol is critical to avoiding spread of infection among hospitalized patients and health care workers.

The standard guidelines for infection prevention include good hand hygiene, universal blood and blood fluids precautions, cleansing and disinfection, sterilization of equipment/instruments, surfaces decontamination, correct use of disinfectants, aseptic techniques, safe disposal of wastes, sharps, handling soiled linen and patient isolation [4]. Other measures that have been incorporated into CDC guidelines include personal health \& safety education, placement evaluation, immunization programs such as screening for hepatitis $\mathrm{B}$ and $\mathrm{C}$, management of health care worker's illnesses and exposure as well as post exposure prophylaxis [4].

A variety of challenges face infection prevention and control in healthcare institutions. This was evidenced by the disparity in knowledge, attitudes, practice and compliance by health care workers [5, and [2]. Studies had shown that poor decontamination of instruments and ineffective infection prevention practices and control often led to outbreaks of nosocomial infections $[6 ; 7]$.

Therefore, the study was important to establish whether there were any gaps in compliance with infection prevention in order to take corrective interventions by the hospital administration.

\section{Problem Statement}

Nosocomial infections often occur in hospitalized patients due to gaps in infection prevention and control practices irrespective of the types of institution. Studies have shown that the incidence of hospital acquired infections vary between $6-15 \%$ in all patient admissions [8]. In Kenya, serious concerns have been raised on infection prevention practices among health care workers due to lack of regular updates on current infection prevention practices. Studies have shown poor decontamination of instruments and ineffective infection prevention practices often lead to outbreaks of nosocomial infections [6;7].

Lack of compliance with infection prevention and control among health care workers has a number of consequences including increased bed occupancy and straining the scarce hospital resources. Furthermore, nosocomial infections present a serious cause for concern due to attendant morbidity and potential mortality. Therefore, preventing nosocomial infections among hospitalized patients is of critical importance for all health care workers. The study aimed at evaluating compliance with infection prevention and control practices among health care workers at a level four hospital in Kenya.

\section{Study Objectives}

The objectives of the study were to:-

1. Identify existing infection prevention and control policy guidelines

2. Evaluate the implementation of infection prevention and control measures

3. Identify the barriers to compliance with infection prevention and control measures

\section{Research Methodology}

The study used a cross-sectional descriptive design. Data collection methods included questionnaire, record review and focus group discussion. The questionnaire was developed by the researchers and was self-administered.

The sample comprised 168 respondents who included nurses, clinical officers, laboratory technologists and cleaners. Purposive sampling was used to select the health care workers who participated in the study.

Record review was done for patients' admission records of months of May and September 2010 which were randomly selected. Consequently, systematic random sampling was used to select every $10^{\text {th }}$ patient record from 1,500 admission records during the two months. One hundred and fifty (150) files were sampled and analyzed for recorded nonsocial infections.

Focus Group discussion members were purposively selected and represented medicine, surgery and maternity departments of the hospital.

Data collected was coded verified and cleaned to eliminate any errors. Data was then analyzed by the Statistical Package for Social Sciences (SPSS-version 17)

\section{Results}

All 168 respondents who participated in the study returned the questionnaire and which was a response rate of $100 \%$.

The highest proportion of respondents were nurses comprising $40.5 \%$ followed by clinicians (19.6\%), support staff $(17.9 \%)$, casual workers $(13.7 \%)$, and laboratory staff $(8.3 \%)$.

\subsection{Written IPPC Policy Guidelines at the Hospital}

Most (96.6\%) of the nurses agreed that there were written IPPC policy guidelines in the hospital wards as well as $80 \%$ of support staff, $73.9 \%$ of casual workers, $71.4 \%$ of clinicians, and $64.2 \%$ of laboratory staff.

\subsection{Awareness of Types of IPPC Policy Guidelines at the Hospital}

Majority of the respondents were aware of hand washing policy guidelines. These included $98.7 \%$ of nurses, $97.6 \%$ of casuals/others, $92.9 \%$ of laboratory staff, $88.9 \%$ of clinicians 
and $88.6 \%$ of support staff.

Those who were aware of decontamination policy included $94.1 \%$ of nurses, $92.1 \%$ of clinicians, $88 \%$ of casuals/others, $78.6 \%$ of laboratory staff and $71.4 \%$ of support staff. Notably, support staff had the lowest level of awareness on decontamination procedures.

In regard to high level disinfection policy, $82.6 \%$ of casuals workers, $82.4 \%$ of nurses, $82.1 \%$ of clinicians, $78.6 \%$ of laboratory staff and (62.9\%) of support staff indicated awareness. A significant number of the respondents agreed that that they were aware of written sterilization policies. These were $92.9 \%$ of clinicians, $89.7 \%$ of nurses, $87 \%$ of casuals, $80 \%$ of support staff, and $78.6 \%$ of laboratory staff.

Furthermore, $87 \%$ of nurses, $80.9 \%$ casuals, $78.6 \%$ of laboratory staff and clinicians and $60 \%$ of support staff were aware of infection prevention standard procedures. Similarly, $100 \%$ of casual workers, $88.2 \%$ of nurses, $85.7 \%$ of laboratory staff, $80 \%$ of support staff and $71.4 \%$ of clinicians agreed on the awareness of hospital waste management policies.

\subsection{Knowledge on the IPPC Policy Guidelines}

Majority of respondents agreed that IPPC policy guidelines were available in their departments/units. These were $86.8 \%$ of nurses, $82.6 \%$ of casual workers, $60 \%$ of clinicians, $57.1 \%$ of laboratory staff and $54.3 \%$ of support staff.

As shown in Figure 1, there was an established IPPC committee at the hospital.

Nevertheless, $60.9 \%$ of casual workers, $55.6 \%$ of nurses, $37.1 \%$ of support staff, $28.6 \%$ of laboratory staff, $20 \%$ of clinicians agreed that IPPC committee was not active. Furthermore, $64.3 \%$ of laboratory staff, $61.8 \%$ nurses, $52.2 \%$ casual workers and $42.9 \%$ of clinicians and support staff indicated that the existing IPPC policy guidelines were adequately implemented.

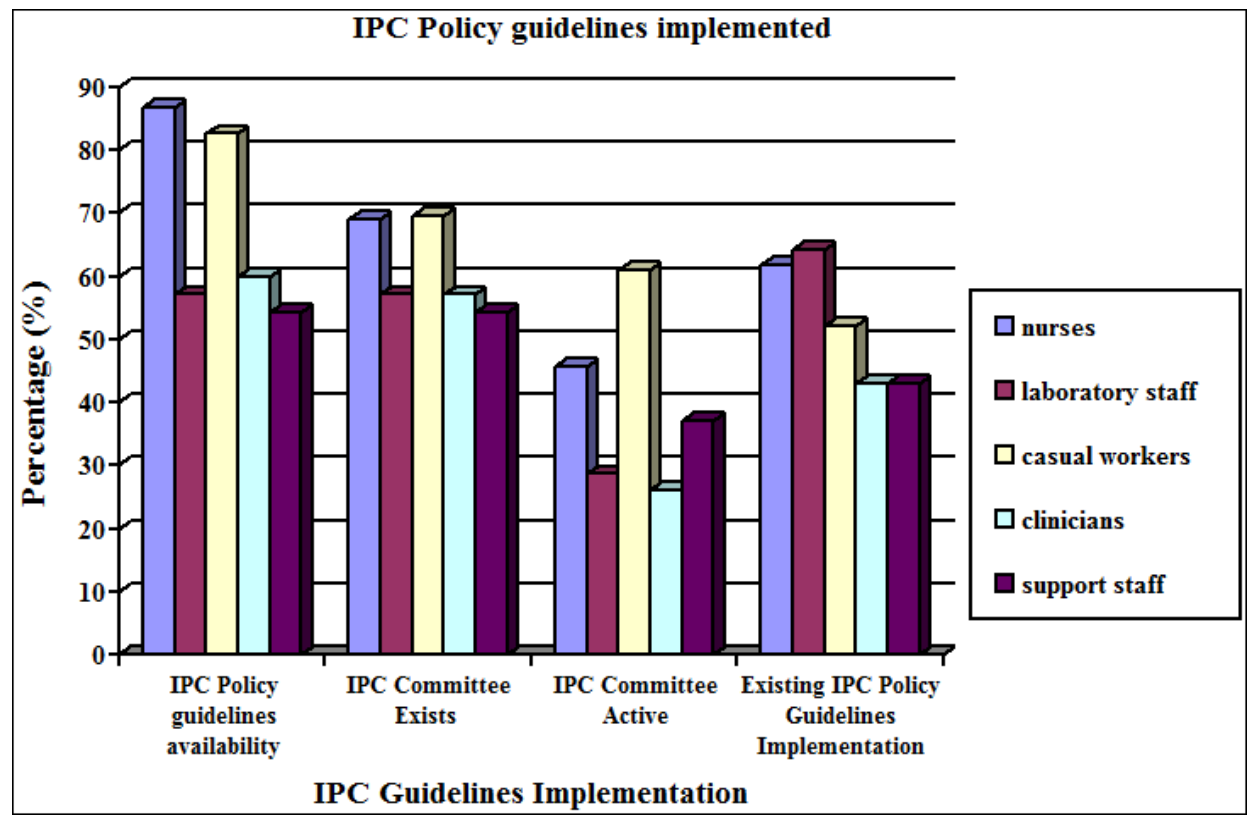

Figure 1. IPPC policy guidelines availability and IPPC committee

\subsection{Compliance with Infection Prevention and Control}

Hand hygiene was frequently done using with soap and water or antiseptic solution. This was confirmed by $91.3 \%$ of casual workers, $75 \%$ of nurses, $71.4 \%$ laboratory staff as well as support staff and $57.1 \%$ of clinicians.

Majority of HCWs agreed they cleaned their hands with sodium hypochlorite solution rather than $70 \%$ ethanol solution. These were $94.7 \%$ of nurses, $74.3 \%$ clinicians, $73 \%$ of casuals, $21.4 \%$ of laboratory staff, and $5.7 \%$ of support staff. While hand hygiene was practiced by HCWs, not everyone washed hands after removing gloves. According to focus group discussion, hand hygiene products commonly used were bar soap and toilet soap under running water though these were not always available.

\subsection{Use of Gloves and Protective Gear}

As shown in Figure 2, majority of the respondents used gloves and other protective gear when carrying out procedures and handling patients. These included $95.7 \%$ of casual workers, $91.2 \%$ of nurses, $78.6 \%$ of clinicians, $78 \%$ of laboratory staff and $74.3 \%$ of support staff. Protective gear such as face mask and plastic eye glasses were inconsistently used by HCWs as shown as by $71.4 \%$ of laboratory staff, $69.4 \%$ casual workers, $55.9 \%$ of nurses, $46.7 \%$ of support staff and $46.4 \%$ of clinicians during provision of patient care. 


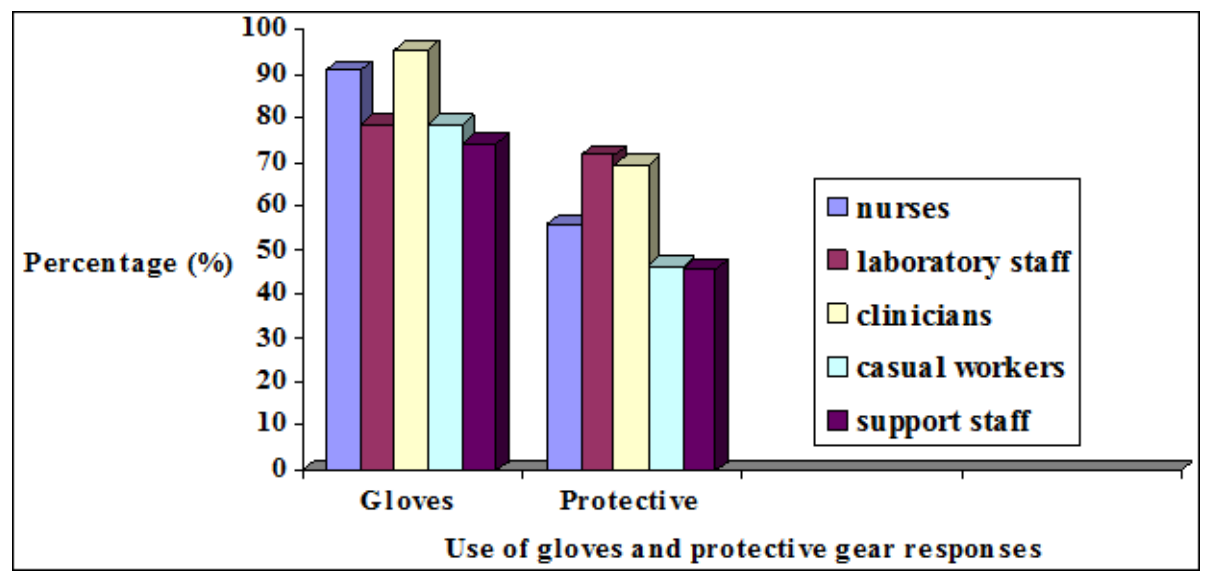

Figure 2. Use of gloves and protective gear when giving patient care

\subsection{Decontamination Procedures}

Majority of the HCWs stated that decontamination of equipment /instruments was done. These were $91.3 \%$ of casual workers, $88.2 \%$ of nurses, $78.2 \%$ of laboratory staff as well as clinicians, and $75.7 \%$ of support staff. Jik ${ }^{\circledR}$ solution was used to decontaminate equipment/ instruments. Focus group discussion responded that decontamination of instruments was carried out though with less than adequate number of buckets.

\subsection{Incidence of Nosocomial Infection}

From the 150 patients' files that were reviewed, a total of 10 nosocomial infection incidents accounted for $6.7 \%$ incidence at the hospital.

\subsection{Barriers to Compliance with Infection Prevention and Control}

Majority of the respondents indicated that here were adequate resources for IPPC implementation. These included $89.5 \%$ of nurses and $71.4 \%$ of laboratory staff who stated that gloves were available and they were being used affectively. Similarly, $68.6 \%$ of support staff and $60.9 \%$ of casual workers stated that gowns were adequate and they were being used effectively. Sixty three point nine percent $(63.9 \%)$ of clinicians and $60.9 \%$ of casuals who rated the lowest agreed that there were adequate face masks. Sixty nine point six percent $(69.6 \%)$ of clinicians and $34.4 \%$ nurses agreed that there were adequate boots. Eighty percent (80\%) of the respondents agreed that there were adequate theatre gowns.

\subsection{Water Shortage}

Eighty six point eight percent $(86.8 \%) 27.9 \%$ of nurses, $(42.9 \%)$ of support staff as well as laboratory, $35.2 \%$ of clinicians and $34.8 \%$ of casual workers indicated that there was frequent water shortage in the hospital. Sixty seven point one $(67.1 \%)$ of laboratory staff, $43.5 \%$ of casual workers and $38.2 \%$ of nurses stated that the sinks did not always have running tap water. The water supply was obtained from the municipal water services. However, alternative sources were the borehole and rainwater were unreliable.

\subsection{Barrier Nursing Technique}

The respondents indicated that barrier nursing technique was practiced for highly infectious diseases including isolation. These were $60 \%$ of support staff, $46.4 \%$ of clinicians, $44.1 \%$ of nurses, $42.9 \%$ of laboratory staff and $39.1 \%$ of casual workers. For barrier nursing, there was use of protective gear to prevent transmission of disease of diseases such as yellow fever, hepatitis B, and measles.

\section{Discussion}

The results showed that most $(98.7 \%)$ of HCWs were aware of various policy guidelines. The high levels of awareness among HCWs at the hospital were likely to support implementation of the policy guidelines. Nurses seemed to be most aware of all elements of standard procedures. This could be explained by the fact that they were constantly in contact with patients and took more selfpreventive precautions compared to other health workers. The standards and guidelines were expected to protect and improve health care by enhancing the quality of care in the health care settings [9].

While the Infection Prevention and Control Committee existed at the hospital but it was found to be inactive. Among the possible reasons for its inactivity was the perception of the HCWs and hospital administration that IPPC committee's role was limited to formulation of policies. This apparent misunderstanding of the role of the IPPC committee potentially led to inadequate support and ultimately poor performance. The IPPC committee plays a key role in monitoring and evaluation feedback and training of HCWs. It is desirable for the hospital management to support the IPPC committee with adequate resources and re-activate it. A fully functional IPPC committee provides a forum for multidisciplinary input, cooperation and information sharing. The committee should act as a liaison between departments responsible for patient care and support department [10].

Hand washing among HCWs at the Hospital was average especially among nurses and clinicians who handled different 
patients frequently. Surprisingly, only $57 \%$ of clinicians washed hands compared to $91 \%$ casual workers. This may be due to attitude which should be addressed by regular updates by continuing education. Preferably, the compliance should be highest among nurses, clinicians and laboratory staff who spend most of their time with the patients and supervise others on implementation of IPPC policies. It may be helpful if hand sanitizers were placed strategically in the corridors leading to the hospital wards. This encourages both workers and visitors to sanitize their hands, thus limiting the chances of spreading of infection.

Hand washing is a major component of standard precautions and one of most effective methods of preventing transmission of pathogens associated with hospital care [11; 12]. The finding of the study concurs with [11; 13] who observed that hand washing was done appropriately only in $57 \%$ of occasions. This was lower than the rates of selfreported hand washing practices in which $86 \%$ of nurses indicated they washed their hands regularly. The proportion of nurses washing hands between tasks was $15.5 \%$ and between patients was at $20 \%$ implying a potentially high risk of cross infection. The role of hand washing in the spread of infections cannot be underestimated since the practice is still inadequate in many countries and health care settings. A related study in the Democratic Republic of Congo found the rate of hand washing among nurses was $9 \%$ and that the most experienced HCWs practiced hand washing the least which may be attributed to resistance to change.

While there were challenges to infection prevention implementation, the occurrence of nosocomial infection was $6.7 \%$. This is comparable to a study by [8] who found that the rate of hospital acquired infections varied between $6-15 \%$ of all hospital admissions. Among factors that could contribute to cross infection are lack of IPPC committee and surveillance team to provide strategy, feedback and continuing education. Standard precaution is the personal practice of using barriers; so that there is no contact with any body fluids, such as blood, secretions, regardless of whether or not the clinicians is able to see blood, non-intact skin or mucus membranes [7]. However, gaps noted by this study were poor documentation and incomplete patient information in the records. The hospital administration needed to develop a method for clinical audit to reduce the gaps to enhance proper documentation and record keeping.

Overall compliance level to IPPC seemed to be average with a mean score of $60.8 \%$ for the hospital. Adequately implemented IPPC policy guidelines were hand washing, decontamination, sterilization (autoclaving), and waste management. Inadequately implemented IPPC policy guidelines were high level disinfection, standard procedure and house-keeping.

The findings showed water shortage in the hospital at the time of the study. To the contrary, a health facility should have adequate supply of safe water for all health activities [10]. Water shortage is an issue requiring urgent attention. Shortage of water hinders service delivery and reduces compliance with IPPC with serious consequences to the patients, health workers and the environment. It is critically important for the hospital to urgently identify an alternative and reliable supply of water to the institution.

Barrier nursing was found to be deficient and seemed to be given inadequate attention. Inadequate use of barrier nursing poses a serious risk for spread of preventable by applying proper techniques. In barrier nursing, hospital personnel wear gowns handling certain patients, hand washing with antiseptic solution between patients and after patient contact, use of hand sanitizers, and disinfection of objects contaminated by patients [14].

Based on results from the focused group discussion, there was adequate implementation of IPPC though there were some challenges experienced. These included inadequate supplies, shortage of staff, high workload and low resources. Some respondents did not view physical constraints within the ward environment such as bed sharing and inadequate spacing hampered infection control and safety. These factors have been cited in similar studies [15] by Also, [11] stated that the most frequently cited reasons for failure to practice effective infection control was lack of supplies, work load and task allocation. For effective infection control, hospital management should fully support IPPC activities, provide adequate resources, establish functional IPPC committee and develop IPPC continuing professional education program for all HCWs.

\section{Conclusions}

From the foregoing findings and discussions, the following conclusions were made:

- There were written IPPC policy guidelines such as posters in clinical area of which majority of HCWs were aware.

- The IPPC measures were not fully implemented due to inadequate resources and compliance level seemed moderate, with a mean of $60.8 \%$. The Infection Prevention and Control committee existed at the hospital but was inactive.

- There were several barriers to IPPC which hindered compliance including inadequate supplies, frequent water shortage and inactive IPPC committee

- There was incidence of nosocomial infections with an overall rate of $6.7 \%$. However, documentation and record keeping was poor.

\section{Recommendations}

- The Hospital administration should provide copies of IPPC policy Guidelines in all wards/units and ensure effective implementation through constant supervision and adequate supplies.

- The hospital administration should re- activate IPPC committee and conduct regular audits to enhance compliance and implementation of IPPC.

- The hospital administration need to urgently address water shortage and identify an alternative water supply 
source.

- Decontamination and record keeping need to be improved.

\section{References}

[1] Hustins, W. C., Dente, B. M., O’boyle, C., O’Reurke, E.J. \& Goldmann, D. A., (2005). Hospital Infection prevention and control: a model for improving the quality of hospital care, low- and middle - income countries. London: Churchill Livingstone.

[2] Zulu, M. B., \& Chalanda., (2001). Investigation of Infection prevention and control in selected Malawian Hospitals. African Journal of Nursing and Midwifery, 3 (1), 46-50.

[3] Manyele, S. V, Ngonyani, H. A., \& Eliakimu, E. (2008). The status of occupational safety among health care providers in hospitals in Tanzania. Tanzania Journal of health research. 10(3), 159-165.

[4] Centre for Disease Control (CDC)., Kenya MMWR Morbidity weekly report (2009). Introduction and transmission of 2009 pandemic Influenza A. HINI virus Kenya, 58 (41), 1143-6.

[5] Omari, M. A., Malonza, I.1., Bwayo, J. J., Matere. E. M., Mwatha, A. K., \& Ndinya - Achola, J. O., (1997). Pattern of Bacteria infection and antimicrobial susceptibility at Kenyatta National Hospital. East African Medical Journal. 110, 135143

[6] Muchina,P.,W. \& Muchina E.,N. (2009). Transmitting infection: Testing disinfectants used in maternty Units. African Journal of Midwifery and Women's health, 3(1), 12-14
[7] Inyama, H. K., Revathi, g., Musandu, J., \& Odero, T. (2009). The incidence of nosocomial infections: Kenyatta National Hospital- Intensive Care Unit. Kenya Nursing Journal 38 (1), $31-43$

[8] Creedon, A.A., (2005). Healthcare workers hand decontamination, practices with recommendation, practices with recommendation guidelines, Journal of advanced Nursing, 51 (93), 208-216.

[9] MOH (2010). National Standards and Guidelines on Injection Safety and Medical Waste Management. Nairobi: Kenya

[10] Borg, M, A. (2007). IFIC Basic concept of infection control (anon)

[11] Kulei, E. Factors Influencing Infection Control, Prevention and Injection Safety Practices among Nurses at Kenyatta National Hospital medical and surgical wards. Unpublished

[12] Wenzil, R., Edmon, M., Pitte, T., Geddes, A., \& Buluzier J.(1998). A guide to infection control in the hospital, An official publication of international society for infectious diseases. Hamilton, B.C Deckers, Inc

[13] KNH, (2010).WHO Infection Prevention and Control. Infection Control Practitioner. KNH

[14] Wilcox, L., \& Dave, M., (2000). COST OF Hospital Acquired Infections and the value of Infection Control,. Journal of Hospital Infection, 45, 81-84 Manuscript, School of Nursing: Nairobi University

[15] Gammon, J., Heulwen, M. S., Gould, D., (2008). Review of Evidence of Sub-optimal Compliance of Health Care Practioners to Standard Infection Control Precautions. Journal of Clinical Nursing, 17, 157-67 